\title{
Expression profile of Oct-4 lung cancer-specific marker prior and subsequent to a salirasib treatment regime
}

\author{
XIANG AO, JIE ZHOU, HONG LING LIANG, MING JIANG and HONG SHENG LI \\ Department of Thoracic and Breast Oncology, Affiliated Cancer Hospital and Institute of \\ Guangzhou Medical University, Guangzhou, Guangdong 510095, P.R. China
}

Received February 4, 2016; Accepted January 17, 2017

DOI: $10.3892 / \mathrm{ol} .2017 .6868$

\begin{abstract}
Lung cancer is one of the leading types of cancer that lead to mortalities in the male and female populations. The existing lung cancer-specific markers are not able to accurately predict the condition of the disease, and the response of these markers can vary under various pathological conditions. The ability for tumors to regenerate following treatment can be more aggressive, and this may be due to the remaining lung cancer-specific stem cells, which are resistant to chemotherapeutic drugs. Evaluating cancer stem cells under various pathological conditions, as well as prior and subsequent to treatment, can help to increase the understanding of the underlying mechanisms. In the present study, a mouse model with initial and advanced forms of lung cancer was developed using tobacco smoke carcinogen. It was observed from tissue sections that there were many actively dividing cells spread throughout the mouse lung tissue with the initial stages of lung cancer, and these cells aggregated in advanced stages of lung cancer. Furthermore, immunohistochemical staining indicated that there was an increased number of octamer-binding protein 4 (Oct-4)-positive cells present in mouse tissues with advanced stages of the disease compared with tissues without lung cancer or at the initial stages of disease. The cancer stem cell population following salirasib treatment was also investigated in two groups. The mice in the early treatment group were administered with salirasib following 1 month of tumor growth, and the delayed treatment group was treated following 2 months of tumor growth. The number of cancer stem cells was markedly reduced in the early treatment group. However, salirasib failed to have any observable effect in the delayed treatment group. Cancer stem cells were analyzed using the marker
\end{abstract}

Correspondence to: Dr Hong Sheng Li, Department of Thoracic and Breast Oncology, Affiliated Cancer Hospital and Institute of Guangzhou Medical University, 78 Hengzhigang Road, Yuexiu, Guangzhou, Guangdong 510095, P.R. China

E-mail: lihongsheng191@hotmail.com

Key words: octamer-binding protein 4, salirasib, A/J mice, cancer stem cells
Oct-4 to improve an understanding of the proliferative ability of cancer stem cells under various pathological conditions, which may lead to the development of novel cancer therapeutics.

\section{Introduction}

Lung cancer is one of the leading causes of cancer mortality in the United States (1). Early diagnosis of lung cancer is essential; if left untreated the cancer is able to spread and affect nearby organs (2). One of the main causes of lung cancer is the prolonged intake of tobacco smoke (3). Lung cancer can be difficult to diagnose at earlier stages, and a quarter of people exhibit no symptoms even following diagnosis (4). The markers associated with cancer are of great interest to clinicians and enable an improved understanding of tumor biology (5). At present there is no universal marker available to detect a particular type of cancer and using markers for cancer detection has a number of limitations. The expression of cancer markers varies significantly between individuals and false positives are likely to occur (6).

Cancer markers are used to diagnose the stage of disease, assess the response to treatment and predict the prognosis; identifying further markers may be beneficial. The markers associated with lung cancer stem cells are of great interest for the understanding of disease progression prior and subsequent to treatment. Performing an analysis of lung cancer stem cells is an accurate method to determine treatment relapse, as cancer stem cells are not easily eliminated during therapy $(7,8)$. Molecular identification of such lung cancer stem cells may be more valuable in clinical practice as the 5-year patient survival rate for lung cancer is <15\% (9). Currently, although no universal cancer stem cell markers are available to identify individual cancer types, a number of cancer stem cells including prostate, brain and colon are identified using prominin-1 (10). The normal lung stem cells assist in the maintenance of homeostasis upon injury (11) and transform into cancer stem cells following mutation (7).

The markers used for identifying lung cancer stem cells require analysis under various pathological conditions and validation prior and subsequent to normal treatment procedures. The present study focused on these factors to validate the octamer-binding protein 4 (Oct-4) marker in a mouse model. 
A

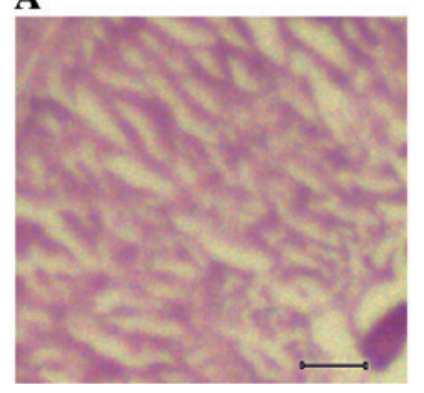

B

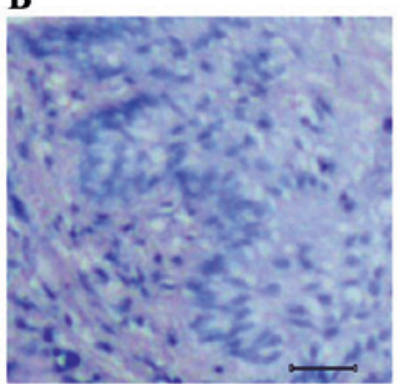

C

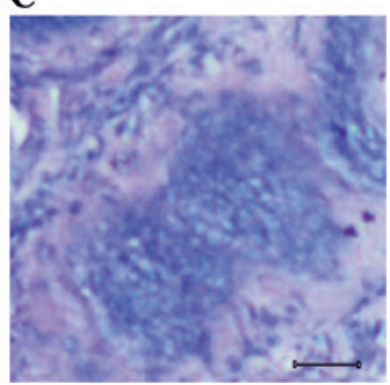

Figure 1. Histological variation between normal, initial and advanced lung cancer tissues. (A) Section of normal lung tissue indicating uniform arrangement of tissue layers. (B) Actively dividing cells spread throughout the tissue layer were visible in the tissue section of a lung tumor at the initial stages of lung cancer. (C) Aggregates of actively dividing cells were visible in the tissue section of a lung tumor at the advanced stages of lung cancer. Scale bar, $50 \mu \mathrm{m}$.

\section{Materials and methods}

Animal experiment. The use of experimental animals throughout the present study was approved by the Institutional Review Board at the Guangzhou Medical University (Guangzhou, Guangdong). A total of $60 \mathrm{~A} / \mathrm{J}$ mice (female, 10 weeks) were randomly chosen for the study, and the mice were divided equally into three groups. The mice were carefully observed for one week in laboratory conditions. The mice were provided with readily available water and food sources. The animals were subjected to regular observations twice a day.

Lung cancer was induced in the mice by exposing the animals to tobacco smoke with a high nicotine content, as previously described by Witschi et al (12). The exposed mice were housed in an animal chamber at $25 \pm 1^{\circ} \mathrm{C}$ and humidity $50 \pm 5 \%$ on a $12 \mathrm{~h}$ light-dark cycle. The mice were exposed to tobacco smoke carcinogen for $7 \mathrm{~h}$ on alternative days. The dosage was continued for a period of 4 months for development of an initial tumor, and mice were similarly exposed for 6 months to develop advanced lung cancer. All mice were given a recovery period of 2 weeks following exposure. The mice were subsequently sacrificed by decapitation for analysis of tumors in the lungs. For treatment, the mice that developed initial and advanced tumors were treated daily with $15 \mathrm{mg} / \mathrm{kg}$ salirasib (Concordia International Corp., Oakville, ON, Canada), which was administered following tumor growth for 1 and 2 months, respectively, via intraperitoneal injection. Control animals were kept in filtered air conditions, without exposure to tobacco smoke.

Immunohistochemistry. The tissue sections were initially fixed in $10 \%$ formalin solution at $42^{\circ} \mathrm{C}$ for 2 days and paraffin embedded. The tissue sections were subsequently subjected to microtome sectioning $(5 \mu \mathrm{m})$. The sections were placed on glass slides, de-paraffinized and rehydrated. The endogenous peroxidase activity was blocked by immersing the sections in freshly prepared $10 \% \mathrm{H}_{2} \mathrm{O}_{2}$ and $10 \%$ methanol in $1 \mathrm{X}$ phosphate-buffered saline (PBS) for $20 \mathrm{~min}$. The sections underwent trypsin treatment $\left(0.1 \%\right.$ trypsin in $\left.0.1 \% \mathrm{CaCl}_{2}\right)$ for $10 \mathrm{~min}$ to cleave the protein crosslinks to assess the antigen and epitope. Nonspecific antigens were blocked using $4 \%$ bovine serum albumin (BSA; Sigma-Aldrich; Merck KgaA, Darmstadt, Germany) for $2 \mathrm{~h}$ at room temperature. The membranes were incubated with an anti-Oct-4 primary antibody (dilution, 1:100; cat no. ab18976; Abcam, Cambridge, UK) overnight at $4^{\circ} \mathrm{C}$. Following incubation, the sections were thoroughly washed with $1 \mathrm{X}$ PBS and incubated with a goat anti-rabbit secondary antibody (dilution, 1:3,000; cat no. ab6721; Abcam) for $1 \mathrm{~h}$ at room temperature. Following washing to prevent non-specific binding, the sections were stained with diaminobenzidine (DAB; cat no. ab64238; Abcam).

Western blot analysis. Tissue samples from mice without lung cancer, initial and advanced stages of the disease were dissected and protein samples were prepared from the cell lysate. Similarly, tissue samples following treatment with salirasib from mice without lung cancer, initial and advanced stages of the disease were taken. Proteins were extracted using $2 X$ SDS sample buffer, and quantified using the Lowry method. The extracted proteins (70 $\mu \mathrm{g} /$ lane) were resolved on a $12 \%$ SDS-PAGE gel, as previously described (13). The protein in the gel was subsequently transferred onto polyvinylidene difluoride membranes. Following blocking with 5\% BSA overnight at $4^{\circ} \mathrm{C}$, the membranes were incubated with the previously described anti-Oct-4 antibody (dilution, 1:400) and a lamin B1 antibody (cat no. ab16048; dilution, 1:500; Abcam) overnight at $4^{\circ} \mathrm{C}$. The membranes were subsequently incubated with the previously described secondary antibody (dilution, $1: 3,000)$ for $1 \mathrm{~h}$ at room temperature. Following washing, the membranes were developed with DAB and imaged and quantified using a Sigma-Aldrich microDOC gel documentation system (cat no. Z692557; Merck KGaA).

\section{Results}

Mice with initial and advanced stages of lung cancer. A total of three groups of mice, each with 20 mice, were selected. The first group served as a control, whilst the second group exhibited the initial stages of lung cancer and the third exhibited advanced lung cancer following exposure to tobacco smoke. The three groups of mice were sacrificed following exposure to tobacco smoke and a recovery period. The lung tissues samples were dissected and subjected to histological sectioning. Clear histological differences were observed between tissue sections of normal, initial and advanced stages of lung cancer, as shown in Fig. 1. The section of normal lung tissue indicated tissue layers with uniform arrangement of cells (Fig. 1A). The tissue sections from mice that were exposed to tobacco smoke carcinogen for 4 months exhibited actively dividing enlarged 
A

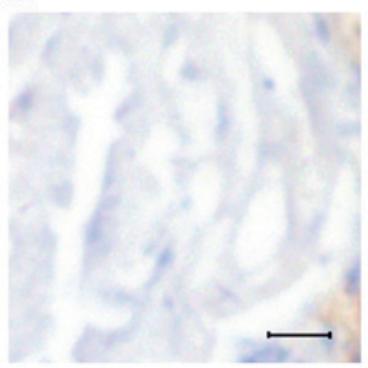

D

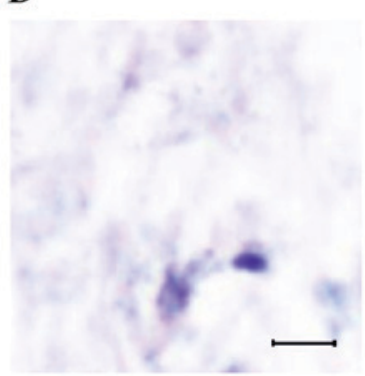

G

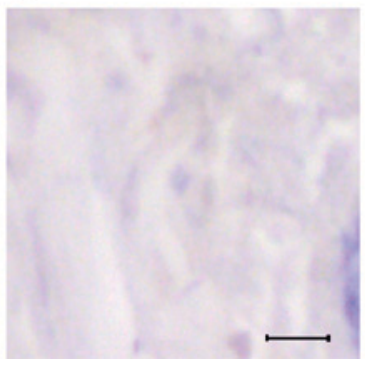

B

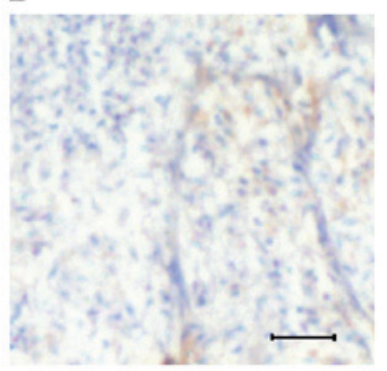

$\mathbf{E}$

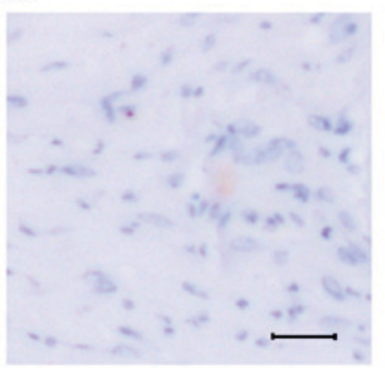

H

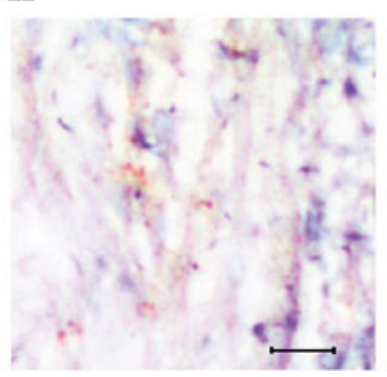

C

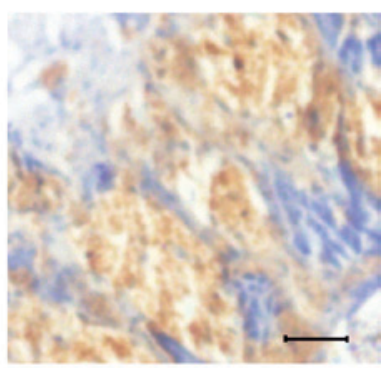

F

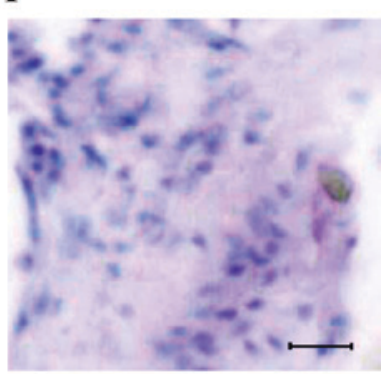

I

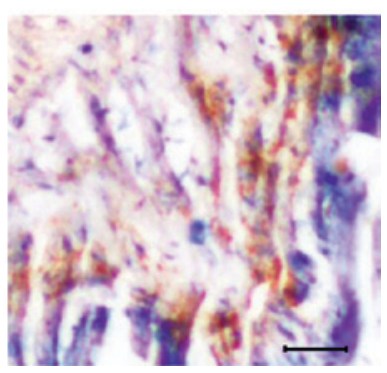

Figure 2. Immunohistological variation is associated with treatment regime. (A) Immunohistochemical staining of normal lung tissue indicated a reduced expression of Oct-4. (B) Increased expression of Oct-4 in lung tissue in the initial stages of lung cancer. (C) Overexpression of Oct-4 in lung tissue at an advanced stage of lung cancer. (D) Oct-4-positive cells in lung tissue in the control group following early treatment with salirasib. (E) Reduced Oct-4 expression in lung tissue in the initial stages of lung cancer following early treatment with salirasib. (F) Absence of cancer stem cells in lung tissue with advanced lung cancer tissue following early treatment with salirasib. (G) Oct-4-positive cells in the control group treated following delayed treatment with salirasib. (H) No reduction in Oct-4 expression in lung tissue in the initial stages of lung cancer following delayed treatment with salirasib. (I) Overexpression of Oct-4 in lung tissue with advanced stages of lung cancer following delayed treatment with salirasib. Scale bar, $50 \mu \mathrm{m}$. Oct-4, octamer-binding protein 4 .

cells that were dispersed throughout the tissue layer (Fig. 1B). Notably, the tissue sections of mice exposed to tobacco smoke carcinogen for 6 months exhibited advanced forms of tumor, with aggregates of cells visible around the center of the tissue (Fig. 1C).

Expression of Oct-4 as a cancer stem cell marker. Although the initial stages of lung cancer following treatment demonstrated marked improvement, it was more difficult to observe changes in the samples with advanced lung cancer. Therefore, a series of experiments was designed to evaluate the changes. Using immunohistochemical techniques, the expression of Oct- 4 was analyzed to validate the pattern of cancer stem cells in normal, initial and advanced stages of lung cancer (Fig. 2). Low Oct-4 expression signals were observed in the normal lung tissue sections, with a number of Oct-4-positive cells (Fig. 2A, D and G). The tissues sections with initial stages of lung tumor exhibited increased Oct-4 expression, and Oct-4-positive cells were scattered throughout the tissue layers (Fig. 2B). In the case of the tissue sections with advanced tumors, overexpression of Oct-4 with a large

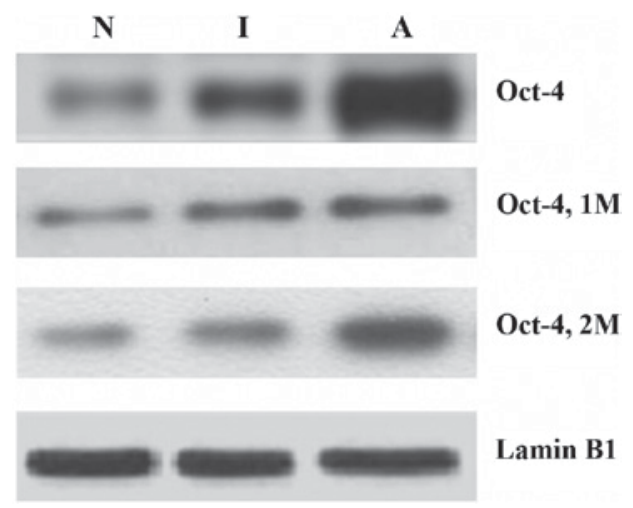

Figure 3. Validation of lung cancer stem cells by western blot analysis with anti-Oct-4 antibody and lamin B1 as a loading control. N, normal lung tissue. I, lung cancer tissue with initial stages of lung cancer. A, lung cancer tissue with advanced lung cancer. $1 \mathrm{M}, 1$ month of treatment with salirasib; $2 \mathrm{M}$, 2 months of treatment with salirasib; Oct-4, octamer-binding protein 4 .

number of Oct-4-positive cells that formed aggregates was observed (Fig. 2C). 
Oct-4 as a marker to assess cancer stem cells prior and subsequent to treatment. In order to study the pathology of lung cancer, cancer stem cells were analysed following treatment with salirasib for 1 month. Mice that were treated early with salirasib, following 1 month of tumor growth, exhibited reduced expression of Oct-4 (Fig. 2E and F). However, mice treated following 2 months of tumor growth displayed increased Oct-4 expression in initial (Fig. 2H) and advanced stages of lung cancer (Fig. 2I). The possibility of eliminating cancer stem cells using salirasib treatment was low in advanced stages of lung cancer.

Analyzing immunohistochemical results using western blotting. Western blot analysis further confirmed the results from immunohistochemical staining as shown in Fig. 3. Similar to the immunohistochemical data, western blotting revealed increased expression of Oct- 4 in advanced lung tumors and slightly reduced expression of Oct-4 following delayed salirasib treatment when compared with initially treated tissue samples.

\section{Discussion}

The persistence of cancer stem cells following treatment is a notable observation in studying tumor recurrence. Cancer stem cells exhibit altered proliferation and differentiation abilities when compared with normal stem cells $(14,15)$. Delaying treatment results in the emergence of advanced lung cancer, which is difficult to control and is associated with poor survival rates with minimal improvement (16).

Chemotherapy and radiation therapy are able to rapidly eliminate proliferative cells, but they are less effective at decreasing the number of cancer stem cells $(17,18)$. Following treatment, the quiescent chemoresistant cancer stem cells proliferate in an aggressive fashion, and this complicates treatment (19). The characterization and identification of cancer stem cells can aid in the correct assessment of disease condition and help to develop novel efficient therapeutics against aggressive forms of cancer (10).

In the present study, initial and advanced stages of lung cancer were successfully induced in mice by exposing the animals to tobacco smoke and altering the exposure time. The tumor at initial stages consisted of actively dividing cells, whereas at advanced tumor stages the actively dividing cells proliferated in an unlimited manner and aggregated. The cell aggregates in advanced stages of cancer is a sign of metastatic development (20). Understanding the underlying molecular mechanisms that regulate cancer stem cell proliferation and the mechanisms involved in the transformation of normal stem cells into cancer stem cells is key in developing novel effective drugs.

From the immunohistochemical data in the present study, it was concluded that salirasib failed to have any observable effect in the delayed treatment group. However, salirasib may have had an effect in the early treatment group by markedly eliminating cancer stem cells. The immunohistochemical results were further validated by western blotting.

Overall, in the present study initial and advanced stages of lung cancer were successfully induced using tobacco smoke carcinogen. Lung tissue sections of initial and advanced stages of lung cancer were differentiated using histological procedures. The number of cancer stem cells was more likely to be markedly reduced when treatment was administered early, (1 month following tumor development), whereas the number of cancer stem cells was not easily reduced when treatment was delayed ( 2 months following tumor treatment).

\section{References}

1. Larsen JE and Minna JD: Molecular biology of lung cancer: Clinical implications. Clinics Chest Med 32: 703-740, 2011.

2. Keshamouni V, Arenberg D and Kalemkerian G: Lung Cancer Metastasis. Springer, New York, NY, 2009.

3. Thun MJ, Hannan LM, Adams-Campbell LL, Boffetta P, Buring JE, Feskanich D, Flanders WD, Jee SH, Katanoda K, Kolonel LN, et al: Lung cancer occurrence in never-smokers: An analysis of 13 cohorts and 22 cancer registry studies. PLoS Med 5: e185, 2008.

4. Beckles MA, Spiro SG, Colice GL and Rudd RM: Initial evaluation of the patient with lung cancer: Symptoms, signs, laboratory tests, and paraneoplastic syndromes. Chest 123 (1 Suppl): 97S-104S, 2003.

5. Mumbarkar PP, Raste AS and Ghadge MS: Significance of tumor markers in lung cancer. Indian J Clin Biochem 21: 173-176, 2006.

6. Thunnissen FB: Sputum examination for early detection of lung cancer. J Clin Pathol 56: 805-810, 2003.

7. Reya T, Morrison SJ, Clarke MF and Weissman IL: Stem cells, cancer, and cancer stem cells. Nature 414: 105-111, 2001.

8. Jordan CT, Guzman ML and Noble M: Cancer stem cells. N Engl J Med 355: 1253-1261, 2006.

9. Jemal A, Siegel R, Ward E, Murray T, Xu J, Smigal C and Thun MJ: Cancer statistics, 2006. CA Cancer J Clin 56: 106-130, 2006.

10. Eramo A, Lotti F, Sette G, Pilozzi E, Biffoni M, Di Virgilio A, Conticello C, Ruco L, Peschle C and De Maria R: Identification and expansion of the tumorigenic lung cancer stem cell population. Cell Death Differ 15: 504-514, 2008.

11. Giangreco A, Groot KR and Janes SM: Lung cancer and lung stem cells: Strange bedfellows? Am J Respir Crit Care Med 175: 547-553, 2007.

12. Witschi H, Espiritu I, Maronpot RR, Pinkerton KE and Jones AD: The carcinogenic potential of the gas phase of environmental tobacco smoke. Carcinogenesis 18: 2035-2042, 1997.

13. Hamidouche Z, Haÿ E, Vaudin P, Charbord P, Schüle R, Marie PJ and Fromigué O: FHL2 mediates dexamethasone-induced mesenchymal cell differentiation into osteoblasts by activating Wnt/beta-catenin signaling-dependent Runx2 expression. FASEB J 22: 3813-3822, 2008.

14. Heppner GH and Miller BE: Tumor heterogeneity: Biological implications and therapeutic consequences. Cancer Metastasis Rev 2: 5-23, 1983.

15. Wicha MS, Liu S and Dontu G: Cancer stem cells: An old idea - a paradigm shift. Cancer Res 66: 1883-1890, 2006.

16. Morgan G, Ward R and Barton M: The contribution of cytotoxic chemotherapy to 5-year survival in adult malignancies. Clin Oncol (R Coll Radiol) 16: 549-560, 2004.

17. Schmidt P, Kopecky C, Hombach A, Zigrino P, Mauch C and Abken H: Eradication of melanomas by targeted elimination of a minor subset of tumor cells. Proc Natl Acad Sci USA 108: 2474-2479, 2011.

18. Dean M, Fojo T and Bates S: Tumour stem cells and drug resistance. Nat Rev Cancer 5: 275-284, 2005.

19. Clark EA, Golub TR, Lander ES and Hynes RO: Genomic analysis of metastasis reveals an essential role for RhoC. Nature 406: $532-535,2000$.

20. Liotta LA, Kleinerman J and Saldel GM: The significance of hematogenous tumor cell clumps in the metastatic process. Cancer Res 36: 889-894, 1976. 